\title{
XXVII. Motions produced by dilute acids on some amalgam surfaces
}

\section{Robert Sabine}

To cite this article: Robert Sabine (1878) XXVII. Motions produced by dilute acids on some amalgam surfaces, Philosophical Magazine Series 5, 6:36, 211-216, DOI: 10.1080/14786447808639498

To link to this article: http://dx.doi.org/10.1080/14786447808639498

曲 Published online: 13 May 2009.

Submit your article to this journal

Џ Article views: 2

Q View related articles $\asymp$ 
usual, by saying "All these motions are rapidly consumed by friction."

It would be possible to determino by observations whether any after-effects of former motions are still present in the ocean. For this purpose it would only be necessary to institute comparative observations of the currents at the most various depths in the central portions of the great equatorial currents and the region of calms. Yet we could not hope to demonstrate small remnants of former motions with a certainty like that with which this is possible in relation to the aftereffect of the former higher temperature of the earth by measurements of subterranean temperatures.

Further, those above-calculated numbers give a hint how remote, at the least, we have to imagine the initial state to have been from the present time, or for how long a period, at the least, we must imagine that the trade-winds have blown in their present extent and strength, to justify the assumption that the present state of motion of the equatorial currents is approximately stationary. For this about 100,000 years are required if we take for a basis a mean sea-depth of 4000 metres and take no account of the damping influence of the continents and islands, which must somowhat lessen that number.

That every initial state, however, finally vanishes, and, from the simple law of distribution laid down, gives place to a stationary one, is evident from the form of the series for $w$, and has, besides, been shown generally for all temperature-problems by K. von der Mübll*.

Giessen, January 5, 1878.

XXVII. Motions produced by Dilute Acids on some Amalgam Surfaces. By Robert Sabinet.

T $N$ the December (1876) Supplementary Number of the 1 Philosophical Magazine I stated an opinion, supported by experimental evidence, that the motions which Erman, Draper, and others have observed in certain electrolytic liquids when in contact with mercury surfaces, and in the circuit of an electric current, are due to displacements caused by oxidation and deoxidation at different points.

In following up the subject since, I have made further experiments, and have noticed some phenomena which are interesting (although perhaps of no practical value), to which, I believe, attention has not hitherto been directed.

* Math. Annalen, ii. p. C48.

+ Communicated by the Author.

$\mathrm{P} 2$ 
Exp. 1. A rather rich amalgam of lead was filtered through a paper funnel into a clean shallow dish, so as to produce as bright a surface as the nature of the amalgam would allow. Then a drop of very dilute nitric acid was carefully placed upon it by means of a pipette. The acid-drop did not lie still (as it would do upon a surface of pure mercury), but set itself at once into a jerky motion. It gradually contracted its area and then suddenly spread out again, then gradually contracted again, and again suddenly spread, the pulsations being irregular. That portion of the amalgam surface which was exposed by the contraction of the drop was very bright and smooth, and appeared to be free from floating particles of foreign metal which roughened the surface at other points.

In a repetition of the experiment with weaker acid, the drop was observed to march bodily over the amalgam surface, going from one side to the other, and sometimes returning.

Dilute sulphuric, hydrochloric, oxalic, and acetic acids behaved similarly to nitric acid, but in somewhat different degrees.

Annalgams of tin, antimony, zinc, and copper behaved similarly to lead amalgam, but also in different degrees.

It was found that, in order to produce these motions, the acid must be sufficiently diluted to avoid the perceptible formation of gas. The extent to which the dilution might be carried appeared to depend upon the richness and nature of the amalgam. Weak motions were observed with the following :-

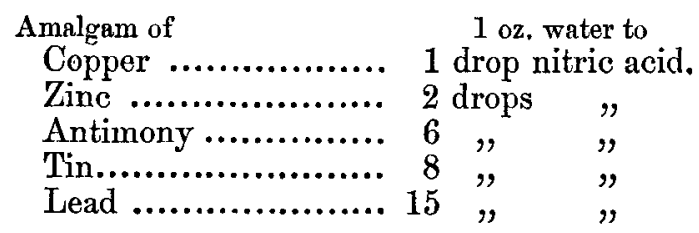

Exp. 2. Instead of the foregoing metals, which in very dilute nitric acid appear to be all positive to mercury, amalgams of platinum, gold, and silver were next tried, these metals being all negative to mercury. On placing the acid-drop on the clean newly filtered surface, no motion whatever was observed. When a trace of positive metal, however, was added, slight motions were observed.

Exp. 3. A dish containing lead amalgam having a drop of dilute nitric acid on its surface was placed under a small glass receiver. The motions of the acid-drop were observed to be irregular, the edge darting out first in one direction and then in another. The air in the receiver was changed by the steady 
injection of oxygen ; and it was observed that the motions immediately became more rapid and, at the same time, more regular all round. When the supply of oxygen was stopped and atmospheric air substituted, the motions resumed their original irregularity.

Exp. 4. The foregoing experiment was repeated by allowing a drop of acid water on the amalgam surface to fall into motion underneath the receiver, and then changing the atmosphere to one of hydrogen. The motions immediately ceased. On letting in the air again, the motions were resumed. In carbonic acid, nitrogen, and coal-gas the motions were instantly arrested; and by covering the amalgam surface under the receiver alternately with one of these gases and with either atmospheric air or oxygen, the motions could be stopped and reestablished at pleasure.

Exp. 5. On a surface of lead amalgam a drop of dilute nitric acid was placed, and the tip of a lead wire suspended in the drop. Between the lead wire and the amalgam was inserted a delicate reflecting galvanometer. By means of an adjustable shunt across the galvanometer, the first current was reduced until the index fell nearly to zero. Then the shunt resistance was increased so as to obtain the greatest sensitiveness, whilst retaining the light point upon the scale. In this position, whilst Mr. McEniry observed the motions of the acid-drop, I observed the motions of the galvanometer, each giving a signal. In this way it was found that each spreading of the drop was accompanied by a slight excursion of the light-point. The direction of the permanent deflection showed that the amalgam surface was positive to the lead wire. The direction of the excursions indicated a slight diminution of this permanent current, or that at the moment of expansion of the aciddrop, the amalgam surface became slightly less electropositive. In the intervals between the expansions, the tendency was for the needle to move in the other direction. Lead is positive to mercury in dilute nitric acid; therefore this reduced electropositiveness may indicate an increased proportion of mercury on the covered surface at the instant of spreading.

The conclusion which I draw from these experiments is, that the motions in question are due to a portion of the surface of the amalgam becoming alternately oxidized by the air outside the acid-drop, and deoxidized by electrolysis in the interior of the drop. The reciprocal play of these two actions is, I venture to suggest, as follows:- When the acid-drop is placed upon the surface of the amalgam of a metal specifically lighter than mercury, it finds the surface to consist of mercury in which are floating innumerable particles of the foreign metal. 
The latter, in rich amalgams, are distinctly visible to the eye, producing a somewhat roughened surface. Electric currents are generated between the mercury and the particles of foreign metal, through the acid. When the foreign metal is positive to mercury, the latter (which by contact with the air is always more or less oxidized) becomes deoxidized underneath the drop of dilute acid and therefore cleaned. The drop has less affinity for the clean than it had for the oxidized surface. Its adhesion is therefore diminished ; and it draws itself together in consequence, leaving a surrounding ring of deoxidized mercury, in which the eye can detect few or no floating particles of foreign metal ruffling the smoothness of the surface. Oxidation of this exposed clean portion of mercury, however, gradually sets in; and when the mercury up to the boundary line of the drop is sufficiently reoxidized, the acid reasserts its affinity for the oxide, and the drop is enabled to spread out again to its original dimensions. Interior deoxidation then goes on again by means of the small surface couples; and the play of alternate contraction by reduced adhesion, due to deoxidation, and spreading by affinity for the oxide formed by the outer atmosphere, is kept up so long as positive metal and acid last.

The contraction due to deoxidation by the current would necessarily be slower than the spreading due to chemical affinity between the acid and oxidized surface.

In explanation of the acid-drop travelling bodily over the amalgam surface, I would suggest that if, from any reason, the oxidation were more energetic at one side of the drop than at the other, it must lose equilibrium and go on travelling in the direction of the more energetic action, because the mercury surface on that side of the drop would be ready oxidized, whilst on the opposite it would be continually left deoxidized.

Supposing that the foregoing explanation is the right one, there are two points well worthy of noting. First, it would follow that the electropositive metal in the amalgam is to some extent electrically independent of the mercury-asserts, in fact, a certain electrical integrity. Secondly, it would be necessary that the electromotive force between the foreign metal and mercury (say lead and mercury) when in very dilute acid must be greater, or at least have a greater decomposing action, when the one metal is in a state of minute subdivision and its particles in intimate electric contact with the other metal, than we should be led to expect from the behaviour of the metals in separate masses.

An analogy appears to exist between this behaviour and the augmentation of chemical affinity of the couples constructed 
by Dr. Gladstone and Mr. Tribe by the deposition of a negative upon a positive metal, described by them at the BritishAssociation Meeting at Bristol in $\mathbf{1 8 7 5}$.

Connected with the same subject, but susceptible probably of a different explanation, is the following experiment:-

On a clean dry surface of rich lead amalgam a drop of strong nitric acid is placed. The acid spreads itself out, the amalgam surface underneath it, in the course of a second or two, assuming a dull leaden-grey colour. Suddenly, with a flash, this colour changes to a whitish tint, which gradually deepens again in colour until, after another second or two, another flash restores the whitish tint. This alternation of colour is sometimes repeated steadily many times in succession during several minutes, until an energetic action at length sets in and gas is evolved.

The change from grey to white is always abrupt; the change from white to grey always gradual.

Instead of employing a vessel with lead amalgam, the flashing was equally well observed with the amalgamated surface of a piece of common sheet-lead. A galvanometer placed in the circuit showed that at the instant of the flash the covered surface became less electropositive.

A quantity of lead amalgam placed in a shallow dish and completely covered with strong nitric acid showed changes of colour as before. At each flash it was observed that the area of the amalgam was suddenly contracted; and it reexpanded during the change of colour from white to grey.

A drop of strong nitric acid placed upon a bright surface of lead attacks it gradually, and produces a dull grey colour which is slightly opalescent.

A drop of strong nitric acid placed upon a bright surface of mercury attacks it energetically, producing white crystals and evolving gas plentifully.

The behaviour of the separate materials suggests an explanation of what may happen in the case of the amalgam, viz. that while the surface is tarning to a grey colour, the nitric acid is attacking lead; and when it suddenly flashes white, the acid is attacking mercury. The strange part of the phenomenon is the apparent passivity of the mercury for some time after placing the acid upon the amalgam. The explanation which appears to me to be the most probable is, that the first contact of the acid with the amalgam surface results in the production of a thin stratum of lead-salt, which floods the mercury surface and tends to keep the nitric acid for a time separated from it. The acid in course of a second or so, however, diffusing through this stratum, commences an attack upon the 
mercury, which is stopped by the stratum of mercury-salt which is formed, and which enables the lead particles to reassert their electropositivenoss and be further operated upon. Then a fresh diffusion ensues and the play is repeated.

\section{The Origin of the Power which causes the Stellar} Radiations. By J АCOB ENNIs*.

T $\mathrm{T}$ is important for scientific men to acquire the habit of 1 regarding all matter as having been diffused equally, or nearly equally, through all space. This is the initial point in our history of creation, the earliest period which we know. From this universal diffusion we can trace, by the operation of well-known forces, the origin of the stars. We can understand how the vast sidereal systems, stellar nebulæ, were formed, and how they must remain stationary in space. We can understand the origin of solar and planetary systems, and how these latter systems are all moving through space with inconceivable velocities. We can learn (as I proved in my paper in the 'Philosophical Magazine' for April 1877) that gravity is the force which imparted their velocities to all the stars and to all stellar systems. And now I am to prove that in this same universal diffusion of all matter wo can behold the reservoir, the illimitable reservoir, of that force which radiates the undulations of heat, light, and actinism from all the stars. I will point out also that, in the condensation from that primitive diffusion, we can behold the origin of those modifications of matter which we call the simple elements. In that condensation also we can see the first manifestations of several of the physical forces, such as electricity, magnetism, cohesion, heat, and light. The three primitive forces, of whose origin we know nothing, are repulsion, chemical force, and gravity.

The diffusion of matter in a gaseous form, by the ordinary repulsive force, is a store of heat; and this store is greater or smaller in proportion to the amount of diffusion. It is analogous to the removal of a pound weight from the surface of the earth : the further it is removed the greater will be the amount of heat produced by its fall. It has been found that when our atmospheric gases expand from one volume to two, their temperature is lowered, and just $144^{\circ} \mathrm{F}$. of heat are absorbed and rendered latent. Every addition of the original volume by expansion renders latent an additional $144^{\circ}$ of heat; that is, so much heat loses its character and form of heat, and it reappears as repulsion or diffusion. When compressed again, that * Communicated by the Author. 